\title{
Radio Planning and Optimization of W-CDMA Systems
}

\author{
Edoardo Amaldi, Antonio Capone, Federico Malucelli, and Francesco Signori \\ Politecnico di Milano, \\ Piazza Leonardo da Vinci 32 , \\ 20133 Milano, Italy \\ \{amaldi, capone, malucell, signori\}@elet.polimi.it
}

\begin{abstract}
Radio planning and optimization of $3 \mathrm{G}$ wireless systems are key issues for service providers which need to control the huge costs involved. In this paper we describe algorithms and a mathematical programming model for locating and configuring base stations aiming at maximizing coverage and minimizing cost. The present model considers both uplink and downlink direction, studied separately in 1,34. The Tabu Search algorithm we propose exploits solutions provided by the uplink model and a simple power control mechanism to drastically reduce the computational time required to find an approximate solution of the mixed uplink and downlink model. Experimental results obtained for realistic instances with voice as well as data traffic are reported and discussed.
\end{abstract}

\section{Introduction}

Most of third generation (3G) wireless access systems are based on W-CDMA (Wideband Code Division Multiple Access), a more flexible but also more complex radio access scheme 10. In recent years these systems have been attracting considerable attention from a technological as well as a scientific point of view. The challenge is to enhance today's mobile telecommunication systems (e.g., GSM) by providing increased capacity, data transmission capabilities and a wide range of new multimedia services.

Due to the peculiarities of W-CDMA, the radio planning problem cannot be subdivided into a coverage problem and a frequency allocation problem like it is the case for planning second generation cellular systems with a TDMA-based access scheme [11]12]. Indeed, in W-CDMA the bandwidth is shared by all active connections and no actual frequency assignment is strictly required. Moreover, the area actually covered by a base station (BS) also depends on the signal quality constraints, usually expressed in terms of Signal-to-Interference Ratio (SIR), and on the traffic distribution [6. Since SIR values depend on emission powers, the specific power control mechanism and the power limitations must be taken into account.

To address the network planning problem for systems with W-CDMA air interface, the following information are usually supposed to be known: i) a set of 
candidate sites where BSs can be installed, ii) a set of possible configurations of each base station (rotation, tilt, height), iii) the traffic distribution estimated by using empirical prediction models and iv) the propagation description based on approximate radio channel models or ray tracing techniques. To take into account SIR constraints, two power control (PC) models are considered: a power-based PC model which assumes that emission powers are adjusted to guarantee a target received power and a SIR-based PC model that assumes that emission powers are adjusted to guarantee a SIR target value to all active links.

In previous work 13] we proposed discrete optimization models and algorithms to support decisions on where to install new BSs and which antenna configuration to select for the uplink direction. Uplink turns out to be more critical than the downlink direction in the presence of symmetrical traffic such as voice calls (see e.g. [10]). Since 3G systems are also intended to provide data services which are expected to have a substantial impact on the downlink direction (e.g., web-browsing) and to yield asymmetrical traffic, the downlink direction has been addressed in [4].

In this paper we describe a complete mathematical programming problem which includes uplink and downlink constraints as well as other relevant issues for BS configuration such as antenna height, tilt, and sectors orientation. Moreover we also consider the constraints due to pilot signals which are emitted by base station at a constant power level to broadcast system information and to allow mobile terminal to select best serving station. The problem formulation considers the SIR-based PC but it can be easily modified to model a power-based PC just assigning a proper value to power variables.

Besides providing a deeper insight into the different aspects of the UMTS base station location and configuration problem, considering and studying separately the uplink and downlink direction models as well as power-based and SIR-based PC mechanisms yields a considerable improvement from the computational point of view.

After describing the model in Section 2, a Tabu Search algorithm which provides good solution in a reasonable amount of time is briefly presented in Section 3. A procedure that allows to reduce the computations needed to determine the emitted powers with a $S I R$-based PC mechanism is also described. In Section 4 results obtained for medium-size realistic instances generated using classical propagation models are reported and discussed. Section 5 contains some concluding remarks.

\section{Model with SIR-Based PC for Uplink and Downlink Directions}

Assume that a set of candidate sites $S=\{1, \ldots, m\}$ where a BS can be installed, is given and that an installation $\operatorname{cost} c_{j}$ is associated with each candidate site (CS) $j, j \in S$. A set of test points (TPs) $I=\{1, \ldots, n\}$ is also given. Each TP $i \in I$ can be considered as a centroid where a given amount of traffic $d_{i}$ (in Erlang) is requested and where a certain level of service (measured in terms 
of $S I R)$ must be guaranteed. The required number of simultaneously active connections for TP $i$, denoted by $u_{i}$, turns out to be a function of the traffic demand, i.e., $u_{i}=\phi\left(d_{i}\right)$. The actual definition of the function $\phi$ is a degree of freedom of the planning process. It can simply correspond to the average number of active connections or to the number of simultaneous connections not exceeded with a given probability $p$. The connection activity factor can also be considered.

The propagation information is supposed to be known, either computed by using prediction tools (e.g. Hata's models or ray tracing) or obtained by actual measurements. In the case of omnidirectional BSs or of directive BSs with a uniform horizontal diagram let $g_{i j}, 0<g_{i j} \leq 1$, be the propagation factor of the radio link between $\mathrm{TP} i, 1 \leq i \leq n$, and a $\mathrm{CS} j, 1 \leq j \leq m$. In the sequel matrix $G=\left[g_{i j}\right], 1 \leq i \leq n, 1 \leq j \leq m$, denotes the gain between $\mathrm{TP} i$ and $\mathrm{BS} j$.

Assuming a SIR-based PC mechanism, for each connection the emission power is adjusted so as to guarantee a signal quality level that is equal to a target value $S I R_{\text {tar }}$ 9].

In the W-CDMA UMTS base station location and configuration problem one wishes to select a subset of candidate sites within the set $S$ where to install directive BSs as well as their configuration, and to assign the TPs to the available BSs so as to maximize the traffic covered and/or minimize the installation costs while taking into account the signal quality requirements in terms of $S I R$ and the power limits on the BSs.

To construct a mixed integer programming overall model, let us define the two classes of variables:

$$
y_{j}=\left\{\begin{array}{l}
1 \text { if a BS is installed in } j \\
0 \text { otherwise }
\end{array}\right.
$$

for $j \in S$ and

$$
x_{i j}=\left\{\begin{array}{l}
1 \text { if } \mathrm{TP} i \text { is assigned to BS } j \\
0 \text { otherwise }
\end{array}\right.
$$

for $i \in I$ and $j \in S$. Suppose we consider directive BSs with three identical 120 degree sectors and with an omnidirectional antenna diagram along the horizontal axis. Let the index set $I_{j}^{\sigma} \subseteq I$ denote the set of all TPs $i$ that fall within the sector $\sigma$ of the BS installed in CS $j$. Obviously, for each $j, I_{j}^{1} \cup I_{j}^{2} \cup I_{j}^{3}=I$ and the index sets $I_{j}^{\sigma}$ with $\sigma=1,2,3$ are disjoint.

Since we wish to maximize the total traffic covered and minimize the total installation cost and those two aspects are clearly contrasting, the following objective function is maximized:

$$
\sum_{i=1}^{n} \sum_{j=1}^{m} u_{i} x_{i j}-\lambda \sum_{j=1}^{m} c_{j} y_{j}
$$

where $\lambda \geq 0$ is a trade-off parameter between maximizing coverage and minimizing costs.

Each TP $i \in I$ can be assigned to at most one BS: $\sum_{j \in S} x_{i j} \leq 1$. 
Note that by restricting the assignment variables $x_{i j}$ to take binary values, it is required that in every feasible solution all active connections must be assigned to a single BS. Moreover, a TP $i$ can be assigned to a CS $j \in S$ only if a BS has been installed in $j: x_{i j} \leq y_{j}$.

We also need to introduce an explicit continuous variable $p_{i}$ to denote either the power emitted by TP $i$ or the power received at each TP $i$ from the BS it is assigned to, depending on which direction (uplink or downlink) is considered. Since the received powers must be nonnegative and there exists an upper bound on the maximum power $P_{\max }$ that a BS or a TP can assign to each connection, we have:

$$
0 \leq p_{i} \leq P_{\max }^{u p}
$$

for uplink and

$$
0 \leq p_{i} \leq \sum_{j \in S} P_{\max }^{\text {down }} g_{i j} x_{i j}
$$

for downlink.

For the downlink direction we also consider the powers of the pilot signals $\hat{p}_{j}$ associated to each BS installed in $j, j \in S$. They are supposed to be constant and equal to: $\hat{p}_{j}=\delta P_{t o t}$, where $\delta$ is equal to 0.15 and $P_{t o t}$ is the total power assigned to each $\mathrm{BS} j$.

Moreover, the limit on the total power that each BS $j$ can emit is accounted for by the following inequality:

$$
\sum_{i \in I} \frac{p_{i}}{g_{i j}} x_{i j}+\hat{p}_{j} \leq P_{t o t} y_{j}
$$

Given the $S I R$-based PC mechanism, for each pair of TP $i$ in $I$ and $\operatorname{CS} j$ in $S$ we consider the following $S I R$ constraints:

$$
\frac{p_{i} g_{i j} x_{i j}}{\sum_{h \in I_{j}^{\sigma_{i j}}} u_{h} g_{h j} \sum_{t=1}^{m} p_{h} x_{h t}-p_{i} g_{i j}+\eta}=S I R_{t a r} x_{i j}
$$

$\alpha\left(\sum_{\substack{k \in I_{j} \sigma_{i j} \\ x_{k j}=1}} u_{k} g_{i j} \frac{p_{k}}{g_{k j}} x_{k j}+\hat{p}_{j}-p_{i}\right)+\sum_{\substack{l \in S \\ l \neq j}} \hat{p}_{l} \sum_{\substack{k \in I_{l} \\ x_{k l}=1}} u_{k} g_{i l} \frac{p_{k}}{g_{k l}} x_{k l}+\eta_{0}$

where for any CS $l$, the index set $I_{l}^{\sigma_{i l}}$ denotes the set of all TPs in $I$ that fall within the sector $\sigma_{i l}$ of the BS installed in $l$, which contains TP $i$. For any single connection between a BS located in CS $j$ and a TP $i$ falling in one of its sectors (denoted by $\sigma_{i j}$ ), the numerators of the left-hand-side terms correspond to the power of the relevant signal emitted by or received at TP $i$ while the denominators amount to the total interference due to all other active connections 
in the system. Thus, constraints (5) and [6] make sure that the $S I R$ of any active connection between $\mathrm{BS} j$ and TP $i$ (i.e., $x_{i j}=1$ ) is equal to $S I R_{\text {tar }}$. Note that, since we consider directive BSs with uniform antennas diagram along the horizontal axis, the propagation gains $g_{i j}$ do only depend on the distance between TP $i$ and CS $j$ and not on the sector $\sigma_{i j}$ in which TP $i$ falls.

Moreover, we have to consider an additional $S I R$ constraint regarding the pilot signal from BS $j$ to TP $i$ :

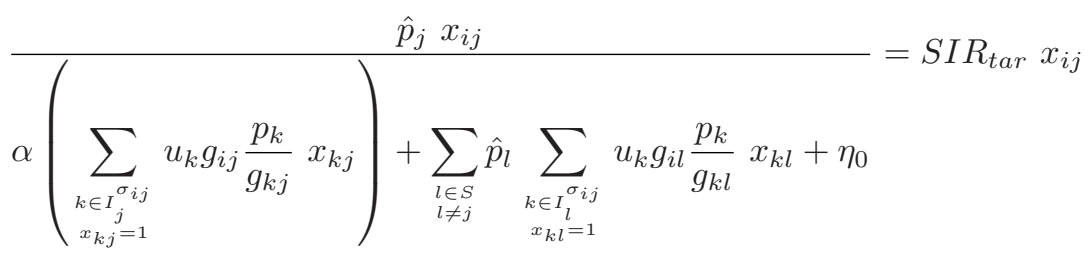

If a power-based PC mechanism is considered, the power emitted from any any $\mathrm{TP} i$ or BS $j$ (depending on which direction is considered) is no longer a continuous variable $p_{i}$, but is adjusted so as to guarantee a received power respectively at $\mathrm{BS} j$ or at $\mathrm{TP} i$ equal to a target value $P_{\text {tar }}$. Therefore, the quality constraints (5, 6) amount to imposing an upper bound on the number of connections that can be assigned to that BS. As new users are added to the system, the $S I R$ values of all the other users decrease until one falls below the lowest acceptable quality level $S I R_{\min }$.

The $S I R$-based PC mechanism is more complex than the power-based one since the power emitted by each BS or by each TP depends on that emitted by all the others, but it is also more efficient since it allows for the use of lower powers. Therefore, from a planning prospective, assuming a power-based PC mechanism instead of a $S I R$-based one leads to a conservative dimensioning which may allocate more radio resources than necessary; moreover, while in uplink direction both mechanisms give good quality results (as shown in section 4), in downlink direction the power-based PC mechanism turns out to be totally inadequate (see [4]).

See also [7] for a discussion of various modelling aspects.

\section{Grasp and Tabu Search Algorithms}

Since even the UMTS BS location problem restricted to uplink direction is NPhard [2, we have developed Greedy Randomized Adaptive Search Procedures (GRASP) and Tabu Search algorithms to find good approximate solutions in a reasonable amount of time.

GRASP is a simple heuristic in which a randomized greedy procedure is applied a predefined number of times and the best solution found is returned. In each run of GRASP, one starts from an empty set of active BSs and at each iteration one randomly selects an available CS (in which to install an additional BS) from a set of available CSs which yield the best improvements in the objective function. 
Tabu Search [8] is a meta-strategy for guiding local search procedures through the solution space of an optimization problem towards good approximate solutions. In our Tabu Search algorithms the following 'moves' are considered to explore the solution space: removing a BS, installing a new BS, removing an existing BS and installing a new one (swap). At each step, a new current solution is obtained by carrying out the best available move even though it may worsen the objective function value. To prevent cycles and to try to escape from local optima, some moves are forbidden for a certain number of iterations (they are added to a Tabu list). The best solution found during the iterations is stored and returned after a predefined maximum number of steps. As initial solution of the Tabu Search algorithm we consider the solution (i.e., the set of active BSs and the assignment of TPs to active BSs) obtained with GRASP.

Looking for approximate solutions of the $S I R$-based model is very intensive computationally even for instances with a moderate number of TPs. Indeed, just computing the transmitted powers corresponding to a given assignment of TPs to BSs involves inverting a $n \times n$ matrix. This operation has to be done each time a single transmitted power has to be changed: clearly, this produces a great computational effort. Moreover, there is no straightforward way of handling power constraints (2) and (3): indeed, if some of the calculated powers $p_{i}$ do not satisfy them, the solution cannot be considered as feasible. To cope with the above-mentioned problems, we adapted a recently proposed iterative method to find the power levels which allows a substantial reduction of computational time [5].

We assume that new users arrive one at a time; before the new arrival, all users achieve their $S I R_{\text {tar }}$. The new user $i$ is given a starting power level high enough to overcome the current interference, i.e. (as an example for uplink direction)

$$
p_{i}=\frac{S I R_{\text {tar }}}{g_{i j}}\left(\sum_{h \in I_{j}^{\sigma_{i j}}} u_{h} g_{h j} \sum_{t=1}^{m} p_{h} x_{h t}-p_{i} g_{i j}+\eta\right)
$$

and the iteration starts only if $p_{i}$ is less than $P_{m a x}^{u p}$. Otherwise, it's no worth trying since the power levels are monotonically increasing during the iterations. After each iteration step, we bound the transmitted powers between 0 and $P_{\text {max }}^{u p}$. If all the links achieve their $S I R_{\text {tar }}$ within maxiter steps (e.g. 5-10), then the new connection is supported. The full algorithm looks as follows:

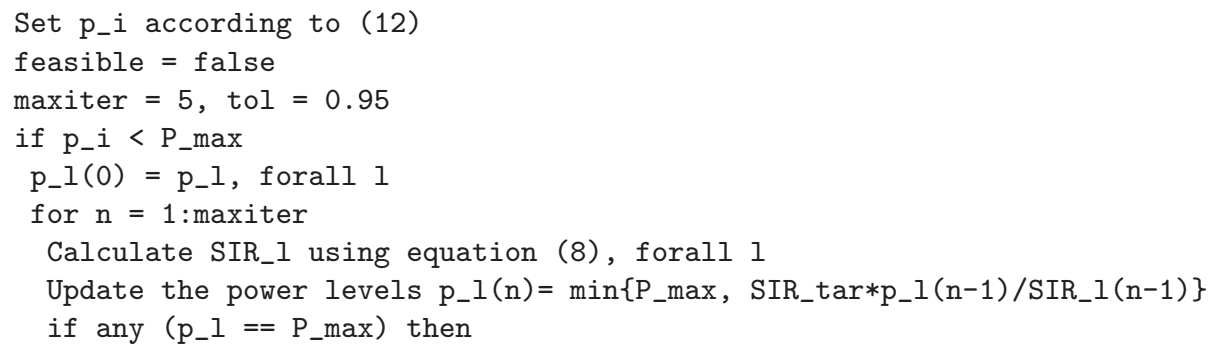




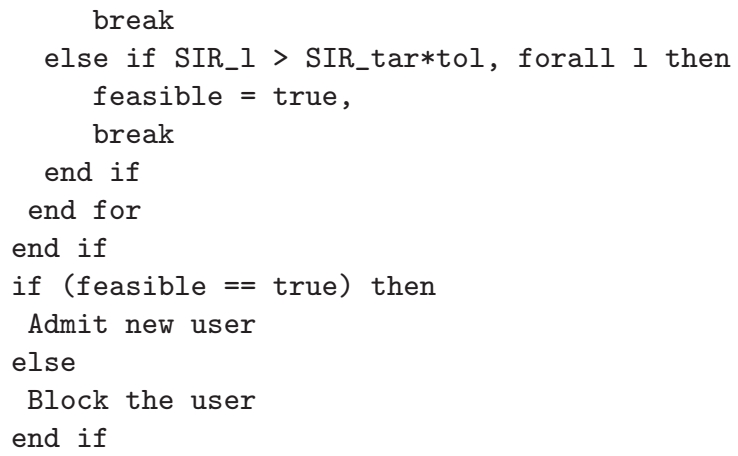

Of course a similar algorithm is implemented for downlink direction.

\section{Some Computational Results}

In previous work 24 we discussed the importance of power control mechanisms in UMTS network planning. Indeed, the $S I R$ depends on the received powers which in turn depend on the transmitted ones: therefore, these powers have to be adjusted by the PC mechanism so as to minimize interference and guarantee quality.

As shown in [2]4, in uplink direction the power-based PC mechanism gives good results, even if it is more conservative than the $S I R$-based one. Yet, the latter needs extremely high computational time, which is in uplink direction almost 10 times higher than the power-based one. Typical results obtained with a single iteration of GRASP and Tabu Search for small-size realistic instances with 390 TPs and 20 CSs are reported in Table1.

As explained in 4], in downlink direction the difference between the results obtained with SIR-based PC mechanism and power-based one becomes more relevant than in uplink direction: with the addition of the pilot signals this difference is still more increased, as shown in Table 2 .

This substantial difference is mainly due to the wide range in which the emitted power levels can vary in downlink direction, thus generating high intracell and inter-cell interference. In the presence of a power-based PC mechanism the emission powers, which are selected to guarantee a $P_{t a r}$ level at the receiving end, are often much higher than needed to yield $S I R$ values equal to $S I R_{\text {min }}$, thus producing high interference levels. On the contrary, with a $S I R$-based PC the emission powers are selected in order to have a signal quality exactly equal to $S I R_{\text {tar }}$, thus keeping the interference levels under limited values; this in turn leads to solutions having excellent coverage with a limited number of BSs.

Due to the above considerations, we focused our attention on the uplink and downlink model with SIR-based PC.

In the first two columns of Table 3 we compare the results obtained with a single iteration of GRASP and with a single iteration of GRASP followed by Tabu Search on realistic small instances including pilot signals and with variable 
Table 1. Results obtained for uplink with the models assuming power-based and SIRbased PC: $n=390, m=20$

\begin{tabular}{|c|c|c|c|}
\hline \multicolumn{2}{|c|}{ SIR-based uplink } & \multicolumn{2}{c|}{ Power-based uplink } \\
\hline Served TPs & \# of BSs & Served TPs & \# of BSs \\
\hline \hline 368 & 7 & 368 & 8 \\
\hline 368 & 7 & 368 & 9 \\
\hline 390 & 9 & 385 & 9 \\
\hline 376 & 7 & 376 & 9 \\
\hline 390 & 7 & 389 & 9 \\
\hline 390 & 8 & 390 & 10 \\
\hline 390 & 7 & 390 & 9 \\
\hline 390 & 7 & 387 & 8 \\
\hline 390 & 8 & 390 & 11 \\
\hline 386 & 7 & 380 & 7 \\
\hline
\end{tabular}

Table 2. Results obtained for downlink with the models assuming power-based and $S I R$-based PC: $n=390, m=20$

\begin{tabular}{|c|c|c|c|}
\hline \multicolumn{2}{|c|}{ SIR-based downlink } & \multicolumn{2}{|c|}{ Power-based downlink } \\
\hline Served TPs & \# of BSs & Served TPs & \# of BSs \\
\hline 385 & 5 & 175 & $\overline{7}$ \\
\hline 383 & 6 & 194 & 9 \\
\hline 390 & 5 & 190 & 10 \\
\hline 390 & 4 & 160 & 8 \\
\hline 390 & 5 & 203 & 11 \\
\hline 390 & 4 & 180 & 9 \\
\hline 390 & 5 & 201 & 11 \\
\hline 390 & 5 & 210 & 10 \\
\hline 389 & 4 & 196 & 10 \\
\hline 390 & 5 & 203 & 9 \\
\hline
\end{tabular}

sector orientations. We consider $m=20 \mathrm{CSs}$ and $n=780$ TPs, equally subdivided in two sets: one producing voice call traffic for uplink direction, the other receiving traffic on downlink direction (see www.elet.polimi.it/res/3Gplanning for some example instances). As expected, the Tabu Search algorithm produces solutions of higher quality than the ones obtained with GRASP: a greater number of users is served with less BSs.

In the third column, solutions produced by an uplink power-based model and subsequently subjected to the uplink and downlink model constraints are shown. These solutions are very similar to the ones directly produced with the complete model (i.e. uplink and downlink together): this is a significant result for at least two reasons. First of all it shows that in a UMTS network the most relevant direction from a planning point of view is without doubt the uplink one (see [4]). This is primarily due to the low intra-cell interference that TPs experiment in the downlink direction because each BS uses orthogonal spreading codes. Moreover, 
Table 3. Results obtained with the model including uplink and downlink SIR-based constraints and with starting solutions provided by the simplified uplink power-based model; section orientation is also considered

\begin{tabular}{|c|c|c|c|c|c|c|c|c|}
\hline \multicolumn{3}{|c|}{$\begin{array}{c}\text { Uplink and downlink } \\
\text { GRASP }\end{array}$} & \multicolumn{3}{c|}{$\begin{array}{c}\text { Uplink and downlink } \\
\text { Tabu search }\end{array}$} & \multicolumn{3}{c|}{ Power-based uplink } \\
\hline TPs dw & TPs up & \# of BS & TPs dw & TPs up & \# of BS & TPs dw & TPs up & \# of BS \\
\hline \hline 383 & 363 & 7 & 390 & 370 & 8 & 384 & 372 & 8 \\
\hline 380 & 369 & 8 & 390 & 390 & 9 & 390 & 389 & 9 \\
\hline 390 & 389 & 11 & 390 & 390 & 8 & 390 & 390 & 9 \\
\hline 390 & 375 & 8 & 390 & 388 & 8 & 390 & 387 & 10 \\
\hline 390 & 390 & 9 & 390 & 390 & 7 & 390 & 390 & 8 \\
\hline 390 & 390 & 8 & 390 & 390 & 7 & 390 & 390 & 9 \\
\hline 390 & 389 & 10 & 390 & 390 & 8 & 390 & 390 & 8 \\
\hline 390 & 390 & 11 & 390 & 390 & 8 & 390 & 390 & 7 \\
\hline 390 & 390 & 8 & 390 & 390 & 7 & 390 & 390 & 8 \\
\hline 390 & 385 & 9 & 390 & 388 & 8 & 390 & 388 & 8 \\
\hline
\end{tabular}

Table 4. Results obtained for downlink assuming SIR-based PC with pilot signal and without pilot signal: $n=390, m=20$

\begin{tabular}{|c|c|c|c|}
\hline \multicolumn{2}{|c|}{$\begin{array}{c}\text { SIR-based downlink } \\
\text { with pilot signal }\end{array}$} & \multicolumn{2}{c|}{$\begin{array}{c}\text { SIR-based downlink } \\
\text { without pilot signal }\end{array}$} \\
\hline Served TPs & \# of BSs & Served TPs & \# of BSs \\
\hline \hline 385 & 5 & 390 & 4 \\
\hline 383 & 6 & 390 & 4 \\
\hline 390 & 5 & 390 & 3 \\
\hline 390 & 4 & 390 & 3 \\
\hline 390 & 5 & 390 & 3 \\
\hline 390 & 4 & 390 & 3 \\
\hline 390 & 5 & 390 & 4 \\
\hline 390 & 5 & 390 & 4 \\
\hline 389 & 4 & 390 & 3 \\
\hline 390 & 5 & 390 & 3 \\
\hline
\end{tabular}

from a computational point of view, the second way of finding good solutions for an uplink and downlink scenario is obviously much less intensive than producing them with the entire model: indeed, the algorithm used for the uplink model with power-based PC is about 50 times faster than the one used for the overall model.

Finally, Table 4 shows the impact of the introduction of pilot signals in $3 \mathrm{G}$ planning: these signals considerably reduce the available power at BSs, thus worsening the quality of the results obtained without them. Moreover, pilot signals themselves have to satisfy some $S I R$-constraints (see 7): again, this produces a degrading of the quality of the results. 


\section{Concluding Remarks}

We have presented a mathematical programming model to optimize the location and configuration of BSs in UMTS networks taking into account both uplink and downlink directions, with pilot signals included, and assuming a $S I R$-based PC mechanism. GRASP and Tabu Search algorithms have been proposed to find good approximate solutions within a reasonable amount of computational time. To make them computationally effective, we adapted a recently proposed iterative method to find the power levels of the signals emitted by TPs or BSs.

Unlike for the uplink model with power-based PC, in downlink the more accurate model with $S I R$-based PC is required. Moreover, the results obtained with the combined model including uplink $S I R$-based as well as downlink $S I R$ based constraints indicate that by starting from the solutions provided by the simplified uplink model with power-based PC, computing times are reduced by a factor of fifty without heavily affecting the solutions' quality. Besides, the introduction of pilot signals obviously worsen the solutions' quality in downlink direction but makes the results more realistic.

Finally, we can assert that the fact of considering and studying separately the uplink and downlink direction models as well as a power-based PC mechanism or a $S I R$-based one has yielded a striking improvement from the computational point of view and has given us a better insight into the overall UMTS BS location and configuration problem.

\section{References}

1. E. Amaldi, A. Capone, and F. Malucelli. Optimizing UMTS radio coverage via base station configuration. In The 13th IEEE International Symposium on Personal, Indoor and Mobile Radio Communications (PIRMC'02), volume 1, pages 315-319, 2002.

2. E. Amaldi, A. Capone, and F. Malucelli. Planning UMTS base station location: Optimization models with power control and algorithms. IEEE Transactions on Wireless Communications, 2002.

3. E. Amaldi, A. Capone, F. Malucelli, and F. Signori. UMTS radio planning: Optimizing base station configuration. In Proceedings of IEEE VTC Fall 2002, volume 2, pages 768-772, 2002.

4. E. Amaldi, A. Capone, F. Malucelli, and F. Signori. Optimization models and algorithms for downlink UMTS radio planning. In Proceedings of IEEE Wireless Communications and Networking Conference (WCNC'03), 2003. To appear.

5. M. Berg. Radio resource management in bunched personal communication systems. Technical report, Kungl Tekniska Hogskolan (KTH), Stockholm, Sweden, 2002.

6. E. Berruto, M. Gudmundson, R. Menolascino, W. Mohr, and M. Pizarroso. Research activities on UMTS radio interface, network architectures, and planning. IEEE Communications Magazine, pages 82-95, February 1998.

7. A. Eisenblatter, A. Fugenschuh, T. Koch, A. Koster, A. Martin, T. Pfender, O. Wegel, and R. Wessaly. Modelling feasible network configurations for UMTS. Technical Report 02-16, Konrad-Zuse-Zentrum fur Informationstechnik (ZIB), Berlin, Germany, 2002. 
8. F. Glover and M. Laguna. Tabu search. Kluwer Publishers, 1997.

9. S.A. Grandhi, J. Zander, and R. Yates. Constrained power control. Journal of Wireless Personal Communications, 1(4), 1995.

10. H. Holma and A. Toskala. WCDMA for UMTS. John Wiley and sons, 2000.

11. C.Y. Lee and H.G. Kang. Cell planning with capacity expansion in mobile communications: A tabu search approach. IEEE Trans. on Vehicular Technology, 49(5):1678-1690, 2000.

12. R. Mathar and Michael Schmeink. Optimal base station positioning and channel assignment for $3 \mathrm{~g}$ mobile networks by integer programming. Annals of Operations Research, 107:225-236, 2001. 